\title{
MICROBIOLOGY
}

\section{Bacterial speech bubbles}

\author{
Stephen C. Winans
}

\begin{abstract}
Many bacteria socialize using diffusible signals. But some of these messages are poorly soluble, so how do they move between bacteria? It seems they can be wrapped up in membrane packages instead.
\end{abstract}

On page 422 of this issue, Mashburn and Whiteley ${ }^{1}$ describe the unexpected convergence of two seemingly unrelated areas of microbiological research: how bacteria talk to their friends, and how they attack their enemies. The authors studied the bacterial pathogen Pseudomonas aeruginosa, which releases a hydrophobic molecule called the 'pseudomonas quinolone signal' (PQS) to send messages to other bacteria of the same species. The surprise is that, rather than being secreted as single molecules, PQS is released in bubble-like 'vesicles' that also contain antibacterial agents and probably toxins aimed at host tissue cells as well.

Various groups of bacteria use diffusible chemicals to signal to their own kind, and this method of communication seems to have evolved independently several times ${ }^{2}$. Pseudomonas aeruginosa is a Gramnegative proteobacterium and, like many proteobacteria, it secretes molecules called acylhomoserine lactones (AHLs). The concentration of these signal molecules increases with cell population density, enabling the bacteria to sense how many of their kind surround them - a phenomenon known as quorum sensing. Whole sets of genes are switched on only at high cell densities, including genes for bioluminescence, DNA transfer, pigment production, and genes required for infecting plants, animals and humans ${ }^{2}$. But AHL molecules are readily broken down by other bacteria, and some AHL signals are poorly soluble in water, so they cannot travel far in an aqueous environment - both factors that would seem to limit their potential as communication signals. PQS, although not an AHL-type signal, is also poorly water-soluble and readily broken down by other bacteria. So how do these molecules manage to spread the word from one bacterium to another?

Mashburn and Whiteley found clues from another bacterial communication system one aimed at enemies rather than friends. Many Gram-negative bacteria release minute vesicles (about $50 \mathrm{~nm}$ in diameter) ${ }^{3}$ from their outer membranes as a means of delivering toxins to host cells and other bacteria (sometimes referred to type VI protein export ${ }^{4}$ ). The vesicles consist of a lipid bilayer surrounding an aqueous core and they can therefore transport lipid-soluble toxins (lipopolysaccharide endotoxin) on their surface and protein toxins in their core. They release their poisonous cargo

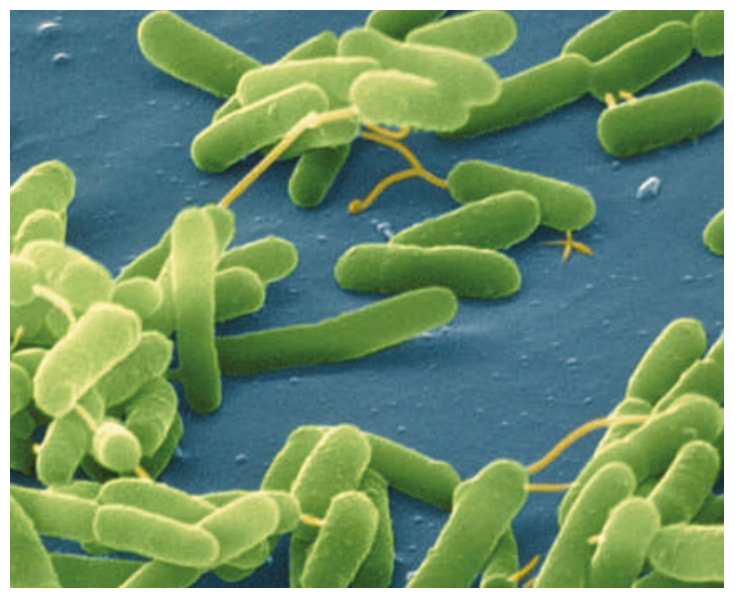

Figure 1 | Friend or foe? Gram-negative proteobacteria such as Pseudomonas aeruginosa communicate with each other by secreting chemical messengers. Mashburn and Whiteley show that they also release such signals in vesicles that contain oxins against other bacteria and the cells of their host.

by fusing with the lipid bilayer of target cells. Mashburn and Whiteley show that these vesicles can also release the PQS signal to other bacteria, thereby sidestepping the problems of poor water-solubility and degradation.

Remarkably, $P$. aeruginosa has three quorumsensing systems that use AHL signals. Apart from the PQS system, there is the LasR/LasI system, which signals using 3-oxo-dodecanoylhomoserine lactone (OdDHL), and the RhlR/RhlI system, which uses butyrylhomoserine lactone (BHL). Of these three signals, OdDHL and PQS are poorly watersoluble, and all three can be degraded by other bacteria. Mashburn and Whiteley found that PQS (but not OdDHL or BHL) is not dispersed between bacteria as single, water-soluble molecules, but rather is concentrated within membrane vesicles, presumably buried within the lipid bilayer.

PQS is one of at least 55 quinolones and quinolines made by $P$. aeruginosa, some of which have potent antibacterial activity. The membrane vesicles, in addition to signalling to kindred $P$. aeruginosa cells, showed potent antibacterial activity against the Gram-positive bacterium Staphylococcus aureus. Most of the antimicrobial activity could be extracted by organic solvents, indicating that toxicity was due to quinolines rather than to the protein component of the vesicles.

The production of the vesicles seems to be regulated by $\mathrm{PQS}$, because when $\mathrm{PQS}$ synthesis was abolished by a mutation in the $p q s H$ gene, vesicle production virtually ceased. Addition of extra PQS restored vesicle formation, even if protein synthesis was blocked by an antibiotic. So, whatever PQS is doing, it is probably happening at the post-translational level, rather than causing alterations in gene expression. Most of the added PQS was found in vesicles, which also contained other quinolones possessing antimicrobial activity. It seems that when PQS is inserted into the outer membrane - whether it was made by that bacterium or added from another source it can destabilize the membrane and spontaneously form vesicles containing itself, other quinolones and presumably

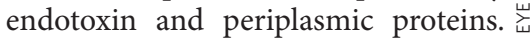
These vesicles can then signal to other pseudomonads, kill unrelated bacteria, and deliver toxic lipopolysaccharides and proteins to host cells.

Pseudomonas aeruginosa is an opportunistic human pathogen that is a particular problem in the lungs of cystic fibrosis patients. Greater understanding about how these bacteria communicate with each other and attack host cells could lead to novel treatment strategies for $P$. aeruginosa infections. The way in which other bacteria generate these ubiquitous membrane vesicles, and what they might contain, is unknown, but most proteobacteria do not synthesize PQS, so other mechanisms must be involved. Moreover, hydrophobic long-chain AHLs were not found in membrane vesicles in this study, so how these signalling molecules diffuse or are transported between bacteria has still to be discovered.

Stephen C. Winans is in the Department of Microbiology, Cornell University, Ithaca,

New York 14853, USA.

e-mail: scw2@cornell.edu

Mashburn, L. M. \& Whiteley, M. Nature 437,422-425 (2005).

2. Waters, C. M. \& Bassler, B. L. Annu. Rev. Cell Dev. Biol. (in the press)

3. Kadurugamuwa, J. L. \& Beveridge, T. J. J. Antimicrob Chemother. 40, 615-621 (1997).

4. Wai, S. N. et al. Cell 115, 25-35 (2003).

\section{Correction}

In the News \& Views article "Cell biology: Powerful curves" by L. Mahadevan and T. J. Mitchison ${ }^{1}$, which discussed a paper published in the same issue ${ }^{2}$, the authors put forward an elastic-sheet model of microtubule structure. Such a model was previously proposed ${ }^{3}$ and applied ${ }^{3-5}$ by Flyvbjerg and colleagues in earlier papers. The authors apologize for the oversight in not citing these earlier quantitative studies.

\footnotetext{
1. Mahadevan, L. \& Mitchison, T. J. Nature $435,895-897$ (2005).

2. Wang, H.-W. \& Nogales, E. Nature 435, 911-915 (2005) 3. Jánosi, I. M., Chrétien, D. \& Flyvbjerg, H. Eur. Biophys. J. 27, 501-513 (1998)

4.Chrétien, D., Jánosi, I. M., Taveau, I. \& Flyvbjerg, H. Cell Struct. Funct. 24, 299-303 (1999).

5. Jánosi, I. M., Chrétien, D. \& Flyvbjerg, H. Biophys. J. 83, $1317-1330$ (2002)
} 